\title{
Collaboration in a multi-user game: impacts of an awareness tool on mutual modeling
}

\author{
N. Nova • T. Wehrle $\cdot$ J. Goslin • Y. Bourquin • \\ P. Dillenbourg
}

Published online: 9 November 2006

(C) Springer Science + Business Media, LLC 2006

\begin{abstract}
This paper presents an experimental research that focuses on collaboration in a multi-player game. The aim of the project is to study the cognitive impacts of awareness tools, i.e., artifacts that allow users of a collaborative system to be aware of what is going on in the joint virtual environment. The focus is on finding an effect on performance as well as on the representation an individual builds of what his partner knows, plans and intends to do (i.e., mutual modeling). We find that using awareness tools has a significant effect by improving task performance. However, the players who were provided with this tool did not show any improvement of their mutual modeling. Further analysis on contrasted groups revealed that there was an effect of the awareness tool on mutual modeling for players who spent a large amount of time using the tool.
\end{abstract}

Keywords Computer supported cooperative work · Awareness · Distributed cognition · Mutual modeling

\footnotetext{
N. Nova $(\bowtie) \cdot$ Y. Bourquin $\cdot$ P. Dillenbourg

Ecole Polytechnique Fédérale de Lausanne, Lausanne, Switzerland

e-mail: nicolas.nova@epfl.ch

Y. Bourquin

e-mail: yvan.bourquin@epfl.ch

P. Dillenbourg

e-mail: pierre.dillenbourg@epfl.ch

T. Wehrle

University of Zürich, Zürich, Switzerland

e-mail: t.wehrle@upsychologie.unizh.ch

J. Goslin

University of Plymouth, Plymouth, UK

e-mail: jeremy@jeremygoslin.com
} 


\section{Introduction}

One of the crux issues in the field of Computer Supported Cooperative Work is the understanding of collaboration [11] as well as the socio-cognitive processes involved when two or more persons are involve in a joint task $[3,4]$. Unlike cooperation in which partners split the work, solve sub-tasks individually and then assemble the partial results into the final output, collaboration is defined by the fact that partners work together [1]. In such a coupled setting, collaboration requires "intersubjectivity": the understanding of another person depends on the assumptions the observer can make about what he's understanding and planning [32]. Carrying out a joint activity depends on this intersubjectivity [23]: a mutual and shared understanding based on the immediate experience of two persons who have only access to their own thoughts and the actions performed by each of the participants. This process we called mutual modeling, is one of the mechanisms of "coupling" that occur during collaboration. It indeed refers to the process by which teammates build and update the shared understanding of the situation as well as presuppositions, beliefs, knowledge and assumptions required to efficiently collaborate. We could expand this mutual modeling definition by saying that it refers to the representation an individual constructs and maintains of what his/her partners does, believes and intends to do.

With the rising use of information technology, work becomes ubiquitous, and multi-user environments allow people to work together from different places at the same time. Nevertheless, achieving intersubjectivity over the distance is tremendously more difficult. That is why scholars came up with the concept of "awareness tools" [12] that allow them to understand the activities, the location and interactions of others. They are supposed to facilitate team collaboration by showing information about presence (is anyone in the workspace?), their identity (who is that?), their location (where is an individual?), their action (what is somebody doing?), and so forth. In the field of Human-Computer Interaction, there have been relatively few occurrences of research concerning the empirical evaluation of awareness tools. Few studies provide data about their use [19] and usability issues about them $[13,14]$.

In this paper, we investigate whether providing people with awareness tools can help them build more accurate mutual models of their partners and hence be more effective. The underlying hypothesis is that maintaining a representation of other's viewpoint explains some of the results of collaborative learning. As a matter of fact, collaboration effects seem to be produced by different kind of collaborative interactions (explanation, argumentation...) that require that people develop a shared understanding of the task. In order to detect misunderstandings and disagreement during the interactions, the subjects must maintain some representation of what their partner understands. It can be postulated that the effort for maintaining a model of the partner contributes itself to the learning process as it forces the learner to reason more deeply on the domain and to perceive the task from a second viewpoint. Now, this raises an old educational issue: if we support mutual modeling with an awareness tool, do we facilitate learning and hence expect higher learning gains or, conversely, do we reduce the cognitive effort and hence decrease the learning gains to be expected? This research, by augmenting mutual modeling with awareness tools, is hence a first step to grasp the cognitive effects of location awareness and mutual modeling. The awareness tools we deal with in this paper are synchronous and concern the location of the partner in the environment.

The following paper will first describe the very concept of awareness with regard to the computational tools that can support it as well as their impacts on group collaboration. The 
second part details the concept of mutual modeling. Then we present our research, the environment and the methodology we used for this experimental research. Then we present the results obtained and discuss and criticize our findings.

\section{A framework of awareness}

\subsection{Introduction}

When people work together in a shared environment (virtual or not), they need information about the action and the intentions of their teammates. This information is critical to a successful collaboration, especially in groupware [5]. This knowledge of others and the result of the participants' interaction and their environment is named "awareness." Dourish and Belloti in [5] have given one of the best-known definitions for awareness: "awareness is an understanding of the activities of others, which provides a context for your own activity." As claimed by [12] awareness is knowledge about the interactions that occur between the teammates and the environment. Maintaining awareness is not the purpose of an activity, it is only used to complete the task. As stated by [27], the term 'awareness' is highly equivocal in the sense that it is used in lots of different ways and is often qualified by many adjectives like for instance 'general awareness' [9], 'workspace awareness' [13] or 'passive awareness' [5]. Definitions indeed range from knowing who is around and then understanding of a social context [5] to the visibility of others actions [15]. To go further, [27] provides a good discussion of the awareness definition problem. We will mainly focus in this paper on the definition of awareness stated by the Calgary research group [12].

According to [12], elements of awareness can be divided into two parts: those related to the present and those related to the past. The former refers to synchronous awareness, the latter to the asynchronous awareness. From Table 1, it can be seen that the most important synchronous awareness information are the elements that answer "who, what, where." The same table can be described for asynchronous awareness and answer to the same question for events that occurred in the past.

\subsection{Techniques for supporting awareness in multi-user environments}

Real-time distributed groupware like shared editors, web portals, whiteboards or multiplayer games allow people who are not in the same place to work together at the same time [6]. These programs provide participants with a shared workspace: a closed

Table 1 Elements of workspace awareness relating to the present [12]

\begin{tabular}{lll}
\hline Category & Element & Specific questions \\
\hline Who & Presence & Is anyone in the workspace? \\
& Identity & Who is participating? Who is that? \\
& Authorship & Who is doing that? \\
What & Action & What are they doing? \\
& Intention & What goal is that action part of? \\
& Artifact & What object are they working on? \\
Where & Location & Where are they working? \\
& Gaze & Where are they looking? \\
& View & Where can they see? \\
& Reach & Where can they reach? \\
\hline
\end{tabular}


environment where the participants can see each other, communicate or manipulate artifacts. In this area, people perform tasks like constructing new artifacts (e.g., architects may draw or design), exploring (finding items), manipulating artifacts, writing texts, etc.

The first multi-users applications were based on the "What You See is What I See" (WYSIWIS) model [30]. They were limited to a small portion of space like a MUD room or a shared whiteboard where all the participants saw exactly the same view of the workspace at all times. While strict WYSIWIS means partners are always looking at the same portion of the workspace, relaxed WYSIWIS lets partners individually look at different portions of the workspace $[10,29]$. That is to say, their viewports into the common space can differ. The relaxations allow people to control their own viewport into the workspace. The problem is that the "Relaxed WYSIWIS" model may create a lack of awareness information; views differ hence users can easily loose track of their partners, where they are and what they are doing.

That is why groupware provide users with Awareness Tools (hereafter AT) to overcome these limitations. AT are employed to "recreate the conditions and clues that allow people to keep up a sense of a workspace awareness" [10]. The information about the participants is gathered, arranged and distributed to the group. Thanks to AT, participants can receive information that can answer the questions presented in Table 1. An example of AT is the radar view: a miniature overview of the workspace that locates the teammates in the virtual environment such as a video game. Another common awareness tool is the status of a person in a chat or their idle time. Telepointers also allow people to see each other's mouse cursor motion when teammates are on the main view (the collaboratively written document for instance).

However, providing awareness raises three big problems: privacy violations, user disruptions and information overload as mentioned in [28]. AT must not violate participants' privacy by showing too many details of other teammates. User disruption is also important since information overload is a growing problem. First, it can distract the user from the essential aspects of his/her work. Second, that information can be irrelevant to the task. And finally, the presentation of information without any explicit user request can possibly disrupt collaboration. An additional problem is the information overload: users should not be bothered by awareness information for every event that occurs. They should also not miss events that are meaningful for their task. In order to avoid those problems, filtering features [2] like a privacy filter [33] or an information filter [25] are often provided with awareness tools.

\subsection{How people use awareness information}

Awareness information may be used for a large variety of purposes during collaboration. Considering workspace awareness (the up-to-the moment information about what is happening in the shared workspace), Gutwin and Greenberg in [12] describe five types of activity aided by the information described in Table 1. First, workspace awareness can be deployed for the management of coupling. Coupling is the degree to which people are working together. The coupling is tight when people are working closely together on an artifact. Loose coupling occurs when people are working somewhat individually, perhaps on different things or different artifacts/parts of the workspace, but where awareness helps them stay aware of what the others are doing. By allowing people to know what a teammate is doing with the appropriate awareness information, they can recognize when the collaboration is possible, when they can present their work to a partner, etc. Second, simplification of communication is a way to employ awareness information: it eases for 
instance referential communication, the process by which a speaker talks about an object to his addressee. "What You See Is What I See" tools have been designed in order to support this process when conversants are not copresent. Instead of describing or citing the object the speaker is referring to, the awareness tool shows the object. The addressee can hence refer to what is being shown. Third, workspace awareness aids how people coordinate actions in collaborative activity. By informing partners about where the teammates are, what they have already done or what they intend to do, it allows people to know when they can collaborate. Fourth, workspace awareness information allows people to understand what is going to be done by their partners. Anticipation and predictions are based on extrapolating forward from present. For example, seeing that a partner is catching an object, one can infer that this artifact is going to be used. Finally, assisting others is a way to use workspace awareness. It can be employed to know if a partner needs help and how. Knowing what he or she has done, where he or she is and what he or she intends to do is useful to help him or her.

\subsection{Examining the existing work about awareness tools impacts}

There is relatively little research concerning the impact of awareness tools. Many papers details the design of AT but relatively few studies discuss their relevance, their effects on the task performance or their collaborative/cognitive impact.

Nevertheless, some researchers $[13,14,17-19]$ examined the usability of the awareness widgets they propose. They use qualitative analysis techniques (observations, interviews, questionnaires) and few quantitative evaluations (measures of the real use). As a consequence, a lot of those studies provide only subjective insights about the AT. The following parts present the issues those authors addressed.

Gutwin et al. in [14] focused on three issues that underlie the usability of awareness tools: the amount and the type of use, the ease of interpreting information and the extent to which AT affects the task completion (simulation of a newspaper distribution). By using qualitative techniques (observation, self-report and interview), they gathered information about the AT used. They found that people really used the awareness widgets provided in their groupware. Although few participants gathered awareness information by asking their partner about where they were and what they were doing, others watched them work thanks to the AT. The participants seemed to be interested in them and expressed their preference for some tools against others. For instance, the view of the limited area around the other user's cursor was never used, whereas the rectangle view of the workspace in miniature were the most used AT. Participants reported that they used the AT in order to understand their partner's activities. Gutwin and his colleagues also found a difference between the ease of interpretation of the various AT they provided in their groupware. For example, in the context of their task, the radar view and the mini view were easier to interpret than scrollbars. In this study, the main problem for the users was to determine the authorship of the actions.

Concerning the extent to which AT affects the task completion, in this experiment, participants claimed that the AT did not distract them. They reported that they were easily able to switch their focus between the workspace and the awareness tools.

In another study, Gutwin and Greenberg [13] compared people's performance when using a simple groupware system and an awareness-enhanced system (with a radar view). The task was to assembly and manipulates simple pipelines in a shared two-dimensional workspace. The AT showed the viewport of the current user and the partner's; it also showed both mice cursors. Thus this radar view provided visual indications of the other 
person's location, the location of his or her cursor, and the motion of objects that he or she moved.

Gutwin's team noticed the following results: the group with AT completed the task more quickly and more efficiently (with less words spoken). Beside, adding AT to a groupware system seemed to improve peoples' satisfaction. They interpreted the fact that the use of an AT increases the performance by claiming that the radar view allowed visual communication. For instance, workspace locations were easier to describe in the AT conditions since the user could see exactly where his partner's view and telepointer were; they could also provide relative directions based on the partner's current location. Gutwin and Greenberg also claimed that the AT, by providing continuous feedback (about piece location for example) and feedthrough enables the players to increase their performance.

Moreover, the awareness information enabled participants to use different and more effective strategies to perform the tasks. Participants used a wide range of verbal and nonverbal methods for indicating locations and pieces. For instance some gave directions based on the other person's current location (e.g., "up and left from where you are") or a description of an object at the location or directions based on a previously identified location. Gutwin and Greenberg noticed differences in strategy use between the two conditions that can be partly attributed to the information available in the two interfaces (simple system and awareness-enhanced system). It seems that pairs in the condition without AT used a wider range of strategies than pairs with AT. Furthermore, the two different groups did not use the same strategy.

This topic has also been tackled by [7]. In their experiments, participants had to collaboratively solve a diagnostic problem by using a computer system. Like [13], they found that groups who used a longitudinal AT completed their task quicker than groups who have no AT. But they established that those who didn't use the AT got closer to the correct solution. They concluded that even though awareness could improve team performance, it might also be detrimental to the team if the information provided are not properly matched to the needs of the specific task. As a matter of fact, in this experiment, AT lead people to pay attention to a wide range of information. Even though the AT contributed to a more efficient division of labor, it led to a cognitive overload. Thus the chronological awareness widget used here did not seem appropriate to the task.

Hubbub [18] is a mobile instant messenger (available on PDAs). It allows people to stay connected as they move. It provides awareness information among distributed groups by giving cues about presence, authorship, identity and activity. Each time a partner becomes active after being idle or offline, a sound plays indicating that someone became active, followed by that partner's Sound ID, so that people can tell who became active without looking. There is also an "activity meter," indicating each partner's level of activity within the last $15 \mathrm{~s}$. Hubbub enables users to meet in opportunistic interactions and then to work over the distance. Like [14], they have shown that the most important information, according to the users and in the context of their task, were activity and location. Presence was also an important feature since people like the Hubbub sounds. One person, who worked exclusively from home said, « sometimes I just like the sounds, just hearing it. It gives me kind of this state of feeling that there's this group and they're working together and you know things are happening 》. Participants used Hubbub to feel more connected to the « dispersed team $»$, that is to say, to develop « a sense of connection with the remote colleagues $\gg$. The authors also aimed to test the usability of the Hubbub sound interface. They found that it was a powerful mechanism for providing background awareness (presence, identity and activity of others), which lots of participants appreciated even though it is a bit disruptive. 
Jang et al. [19] describes a study about the amount and the type of use of an AT in the long-run. The authors stressed the issue of the real use of those awareness features. Unlike [14], their study lasted 13 weeks. They used TeamSCOPE, a web-based collaborative system that allows communication and coordination in dispersed engineering design teams. They found important variations in the frequency of use of the AT and in the distribution of use among partners within the groups. Their findings revealed that only a minority of users (within a group) employed the AT and their use decreased toward the end of the period. These results can be explained by the fact that teams adopt and use groupware differently depending upon specific contexts. As a matter of fact, factors like the availability of alternative supply of awareness information can influence the use of AT.

Ljungstrand and Segerstad [21] explored the extent to which AT influenced the respondents' activities by focusing on how awareness cues of presence affects written message in instant messaging communication. The authors found that awareness of presence is one factor that can influence message composition. Messages sent between lab rooms (distributed) were more related to social coordination (coordinate lunch or coffee break) or to work (《 have you finished exercise 3? 》). Messages sent within the same room seemed to be more mischievous (like funny comments).

Ott and Dillenbourg [26] examined if the use of an AT can modify the grounding process. The authors found that spatial awareness supports grounding by providing people with the contextual cues necessary to refer to objects, that is to say, knowing what the teammate is looking at could reduce referential ambiguity.

Finally, few researchers have explored the perceived value of the AT. Users of AT often express their belief that those widgets had a positive effect on their work [14, 18]. In the evaluation of the Hubbub system [18], in which a sound ID plays when a partner become active after being idle or offline, a female user said that she liked the sounds because it gave her the impression of "being in a group."

Of course, all the awareness tools presented in this section are not comparable since they target many different purposes. Managing coordination in a shared workspace is indeed far from contact facilitation in an instant messenger. Anyhow, they certainly do show the scope of how awareness can be applied in many context, environments and tasks with different scales. The comparisons we drew only lay in the fact that they provide awareness information of some type.

\section{Mutual modeling}

Mutual modeling is one of the collaborative processes that occur when two or more persons carry out a joint activity. We defined it in the introduction as the representation an individual makes of his/her partners' action, strategies and intents. One of the crux issue tackled by social psychologists as well as groupware designers is how an individual can estimate the knowledge, the understanding of a situation and the plans of his/her partner on the basis of the perceived information [32]. This issue is addressed by the attribution theory [16]. Attribution theory is concerned by how people explain things to make sense of the world they live in. More specifically, it focuses on how people try to determine why other persons do what they do. Attribution is an underlying mechanism of collaboration, due to its importance in social interactions. Indeed, collaboration requires that partners take one another's perspective into account during the joint activity.

The attribution process occurs in three steps: perception of action made by the actor, judgment of intention by the observer, and attribution of causes by the observer. People are 
good at estimating the knowledge of others in some domains, using relevant information like gender [8] or social category memberships [20] for instance. However, the judging intentions made by the observers are often biased. [24] showed that people use their own knowledge as the basis for a default model of what other people know.

The issue of estimating others' knowledge within a group is also tackled by the Transactive Memory Theory proposed by [31]. This theory examines the process by which individuals determine who knows what and who knows who knows what. Wegner claims that transactive memory in a group occurs when each member keeps information on who knows what and develops a sense of the group's areas of expertise.

We define the concept of mutual modeling (from now on called MM) as the representation that an individual builds of his/her partner(s): knowledge, goals, strategies, and understanding of the situation, beliefs and plans. In essence, MM results form the attribution processes described previously. Furthermore, MM is more than just transactive memory because it does not only focus on estimating others' knowledge, but also on more "high level" characteristics such as purposes or intentions.

Furthermore, MM is a dynamic representation. Indeed, the initial MM is modified during the achievement of the collaborative activity by all the events found relevant by the partners: others' interaction with the environment, the artifacts, teammates, other's reaction to an action undertaken by a partner, etc.

The link between awareness and mutual modeling has not been studied so much. However, [1] presents a tool to provide awareness about the knowledge that group members have about a certain topic in order to modify the construction and maintenance of shared knowledge. The authors postulate that this knowledge awareness can have positive impacts on mutual modeling and can hence improve group learning.

\section{Delimitation of scope}

\subsection{Motivation}

The aim of this study is to investigate the relevance of awareness tools and their effects on the group performance. Our research question deals with the relationship between an awareness tool and the mutual modeling process. During joint activities, peers maintain a representation of what their partner does, believes, knows and intends to do. The previous part has shown that in order to support fruitful interaction between dispersed teammates, multi-user environments provide AT. Those tools enable users to have information on their peer activities. This should contribute to the mutual modeling (MM) process. However, users need to interpret the information provided by the AT to infer his/her teammates knowledge, strategies or plans. It is possible that AT increases the workload because more information needs to be processed. Indeed, partners could be less effective if there is no resource available to think about the task.

In the context of this experiment, the AT we used is a location awareness tools that indicates the position of the partner in the spatial environment.

The following section describes the environment and the variables we used.

\subsection{The game platform}

Spaceminers is an experimental platform in the form of a video game for running psychological experiments. It has been developed at the Geneva Interaction Lab (University 
of Geneva) by Yvan Bourquin, Jeremy Goslin and Thomas Wehrle. Spaceminers is designed to elicit collaborative behavior. The use of a game metaphor has the advantage that it allows the presentation of complex problem solving tasks in an enjoyable environment, thus maintaining a high level of motivation amongst subjects. However, these statements only hold for subjects that find such games enjoyable, those with little interest in games can fail to engage with the game, finding both the task and the interface difficult and confusing.

The game involves two players in space missions where they have to launch drones in order to collect asteroids full of minerals and bring them to space stations.

As can be seen on Fig. 1 the space is re a grid with planets, asteroids, the spacestation (the circles on the left) and the spaceship explorer. The line shows the trajectory of a launched drone. Here, the direction is modified by the planet's gravity. The purpose of the mission represented here is to collect the largest amount of minerals located in asteroids and to bring them to the space station on the left. The score represents the number of collected minerals docked to the space stations by the two players. The score is influenced by several factors such as : the drone trajectory, the launch speed, the tools' positions (that influence

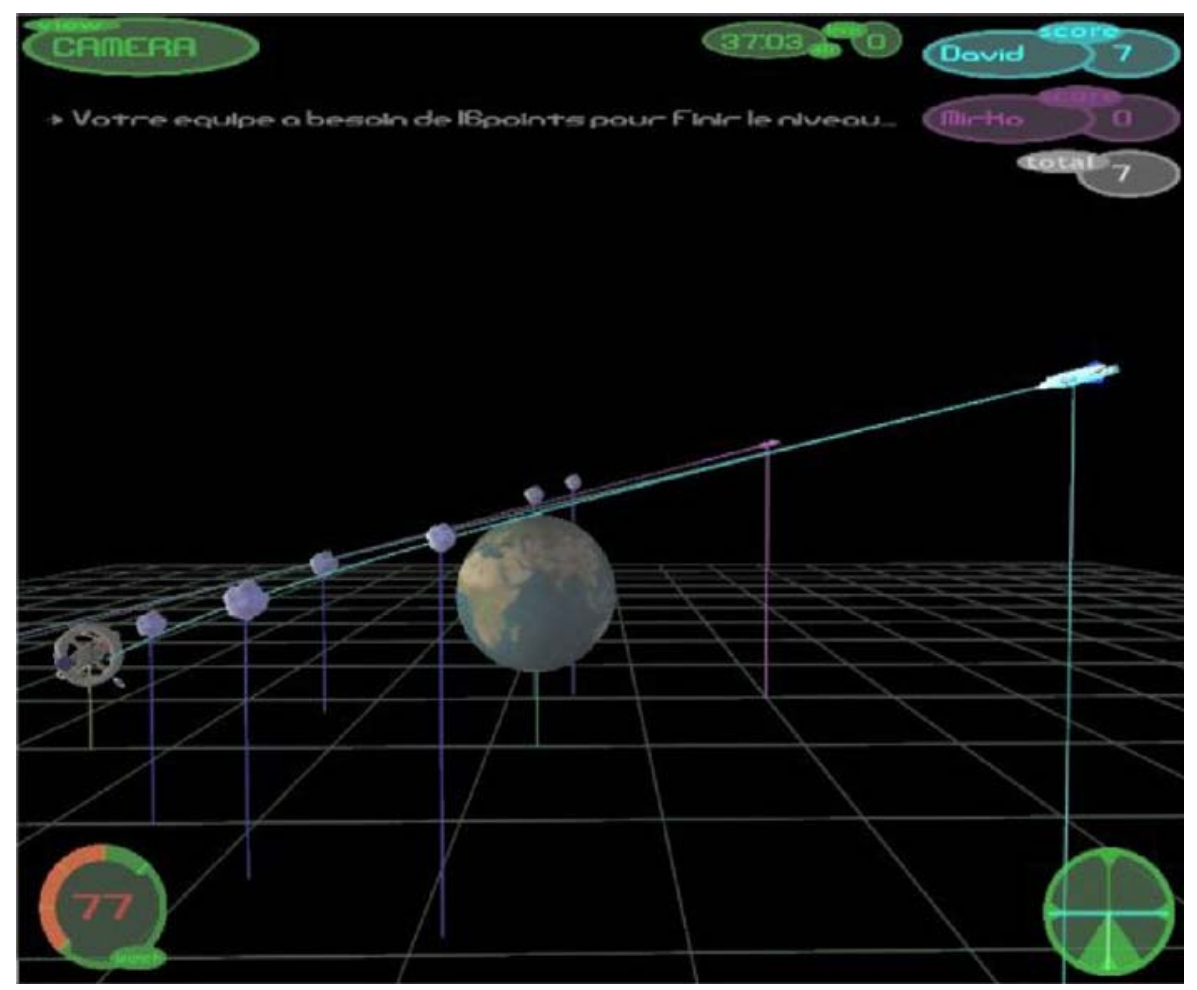

Fig. 1 The game environment made up of a planet (at the center of the picture), asteroids (on the left of the planet), two spaceships (on the right) and a space station (on the left). This kind of view can be seen in the scout mode. The individual scores (for David and Mirko) as well as the group score are located in the upper right-hand corner. The timer and the current game level are just on the left of the score on the top. In the lower left-hand corner is located the launch speed. One can see the compass in the lower right-hand corner. This screenshot depicts the scout view (since we see the spaceship) as indicated in the upper left-hand corner. David (the player who controls the ship) manages to collect asteroids and to dock his drone to the space station. Thus he wins seven points 
the drone trajectory), the number of asteroids in the environment and the planets' positions (that modify the gravity).

Users can play in two modes that correspond to two viewpoints: the explorer mode and the scout mode. They can switch from one mode to another by pressing a key on the joystick.

In the explorer mode, the position of the spaceship is fixed and players launch drones that pass through as many asteroids as possible on their way to the space station. Once the drones are launched players have no control over them. Their trajectory depends on (a) the direction of the explorer, (b) the launch speed of the drone controlled by the player, and (c) on the gravity of planets. The aim of the player, in the explorer mode, is to control the first two variables in order to plan the trajectory of the drones. To change the orientation of the explorer, players can move a crosshair located in the centre of the screen in the explorer view with the joystick. Moreover, using the slider on the joystick can change the launch speed. In the lower left-hand corner, there is a graphical indication of the launch speed. Additionally, different tools dragged into space by players could modify the drone's trajectory.

In the scout mode, players can move their "scout" vehicle around in space by moving the joystick. The scout is very useful to see the space from another viewpoint and to place different artifacts in space. Figure 1 shows the space and the ship seen by the scout. Figure 2 shows the scout as it can be seen in the explorer mode.

To prevent players from being lost in space the interface shows a compass (in the lower right-hand corner) to help players know where they are pointing, the area they can see and whether they are looking up or down. The compass is similar to a normal compass. One line shows you where you are pointing at. The portion indicates the area of space you can see. Finally, the horizontal line shows if you are looking up (if the line is at the top of the compass) or down (if the line is at the top of the compass).

Finally, there is an indication of the score (of the two players and the team) in the upper right-hand corner. The time limit and the game level are also indicated near the score (on the left).

In our experiment, we focused on one kind of awareness tool: the view of the partner's scout and his laser pointer as presented in Fig. 2. By seeing the scout of his partner the player can obtain awareness information about his teammate location and gaze. Thus he
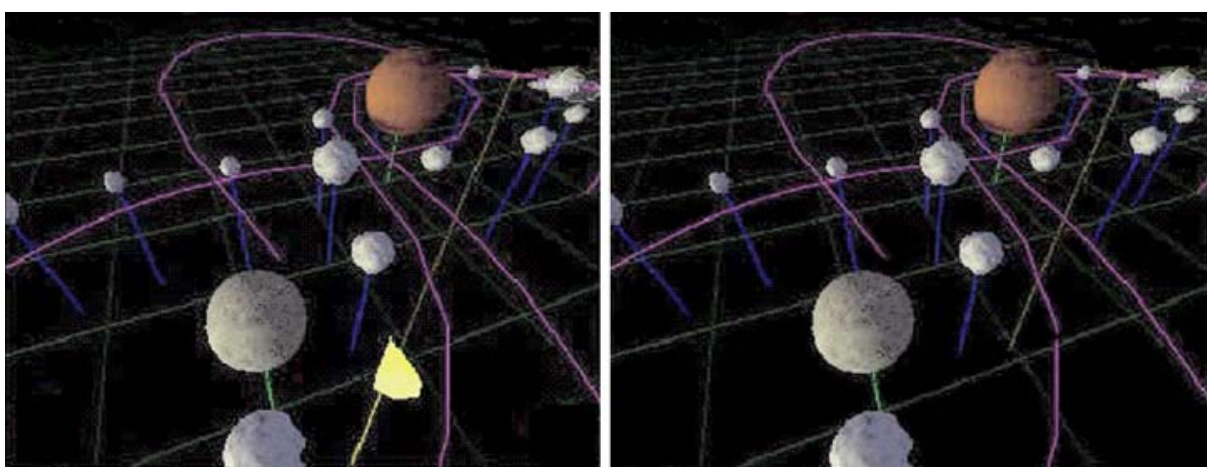

Fig. 2 Presence (figure on the left) or absence of the awareness tool (figure on the right). The view of the scout, represented by a polygon, is basically the awareness tool. This view can only be seen in the explorer mode (from the spaceship). Thanks to this awareness tool represented on the left, the player can know where his partner is and also where he is pointing by seeing the direction of the cone 
could help him to drop spatial objects into space or to adjust the trajectory of the drone the partner intends to launch. The presence or absence of this awareness tool constitutes the experimental condition of the study. We expect players to use this location awareness tool so that they avoid paying attention of their position and space or avoid describing each other's location.

Figure 3 presents the two available spatial objects that can be dropped in space or dragged behind the player's scout: the back hole and the gate. The black hole has a very high gravitational pull: it pulls drones towards it. Gates are stabilized entrances to wormholes in spacetime. If two are placed in space then a gate will transfer a drone from one position in space to another instantaneously. Players see the spatial objects available represented in a toolbox. When someone drops spatial objects in space, the icon is removed from the toolbox and the object stands in space just behind the player's explorer. If he wants to see it, he has to make a rotation.

The spatial objects available depend on the level of the game. On game level one, players are given no objects. On game level two and three, each player has different objects in order to foster collaboration. On game level 2, each player has only one gate. On game level 3, one player has black hole and a gate and the other has only one gate. The procedure of collaborative problem solving consists in the fact that the two players need to negotiate about the position of those objects in space. It is not possible to finish the three levels without talking to each other or deciding where to put a tool. Another collaborative behavior is to help the partner to navigate, to drop an object, to adjust his/her shot as well as role distribution.

Although Spaceminers is a video game, one should not think the task proposed is simple. Playing is hard work for four reasons:

- The 3D navigation is hard. Although the game environment uses conventional 3D representations (like VRML scenes), a few players did not understand the configuration of the world. It could be difficult to perceive the positions of the objects (planets, asteroids, etc.). Two participants (removed for the analysis) were completely lost in space.
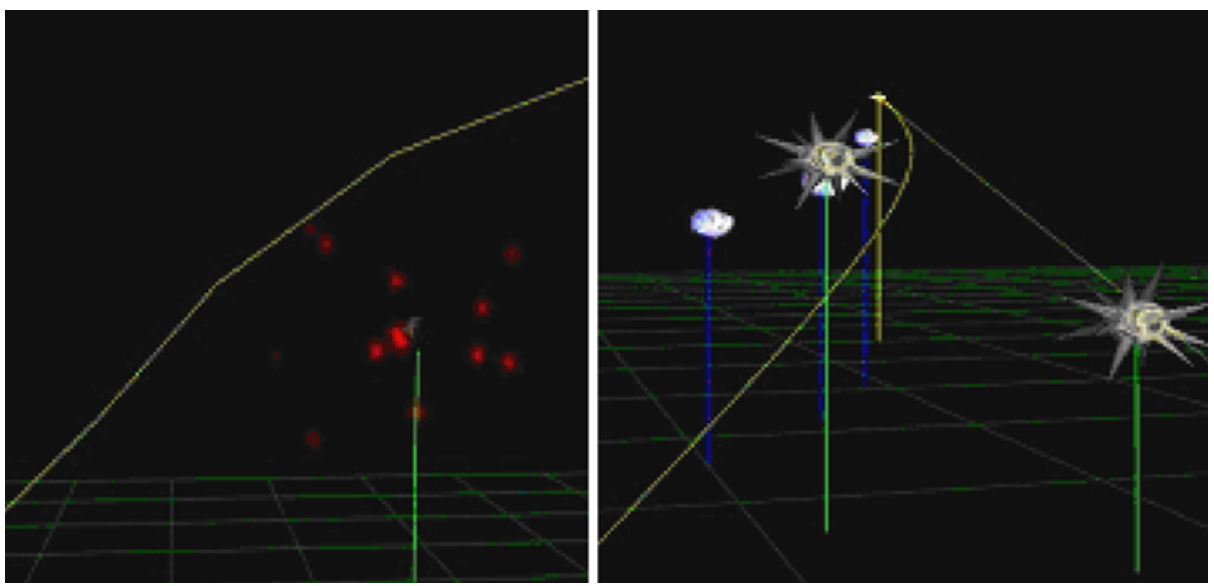

Fig. 3 The two spatial objects: black hole on the left and gates on the right. The black hole pulls the drone; that is why the trajectory is curved. The picture on the right shows that the drone is sent on the first gate (which is on the right), after a while, it will go out of the left gate 
- Ergonomics: the interface is also difficult to use. At the beginning, the participants could be annoyed by the large number of controls on the pad. Beside, the matching between the control and the corresponding actions is sometimes hard. For example, a few players in the pre-experiments did not manage to move their camera. That is why we provided them with a tutorial.

- The task is hard from a cognitive point of view. Players have to understand the goal of Spaceminers and how to reach it. To collect asteroids, they have to control lots of factors cited previously (planets, launch speed, drone trajectory, tools, etc.). Factors like objects positioning is hard to understand and to do.

\subsection{Variables}

The presence or absence of the awareness tool constitutes the experimental condition of the study: it is our independent variable. We used two dependent variables in order to test our hypotheses: task performance and mutual modeling.

Concerning task performance, we used the pairs' combined score: player A's score added to player B's score. Concerning mutual modeling (MM), two different questionnaires (presented in Tables 3 and 4) allowed us to evaluate the value of MM for a pair. First, during the game, players had to answer two multiple-choice questionnaires. Those questionnaires asked them about what they are intending to do at the moment (guiding his partner, trying to understand his strategy, trying to establish a common strategy, adjusting a shoot, etc.). Then, the questionnaires asked each player about what he thinks his partner was intending to do (same propositions as the previous questionnaire). We compared the first answer of a player (about what A is intending to do) to the answer of his partner to the second question (about what B believes $\mathrm{A}$ is doing). Consequently, our evaluation of the MM accuracy is the number of common answers to those two questions. We compared whether A's prediction of B's answer matches with B's actual answer. Thanks to those MM evaluations, we could define different variables presented in Table 2. The mutual modeling indexes we calculated ranged from 0 to 4 .

In game 1, questionnaires are displayed 5 min after the beginning, so that players could be questioned on the actions they are performing at that time and not at the very beginning of a game level. In games 2 and 3, questionnaires are displayed after the first time a player dropped a tool in the environment.

Tables 3 and 4 propose the questions asked in the mutual modeling questionnaires presented in each of the game level.

Table 2 Description of the dependent variables concerning mutual modeling

\begin{tabular}{ll}
\hline Evaluation & Description \\
\hline MMg = MM1 + MM2 + MM3 & $\begin{array}{c}\text { Global mutual modeling evaluation } \\
\text { for the pair } \\
\text { Mutual modeling evaluation for the } \\
\text { pair measured in the first game } \\
\text { MM1 }\end{array}$ \\
Mutual modeling evaluation for the \\
pair measured in the second game \\
Mutual modeling evaluation for the \\
pair measured in the third game
\end{tabular}


Table 3 Questions asked in the first mutual modeling questionnaire in each of the three games
What do you intend to do now?

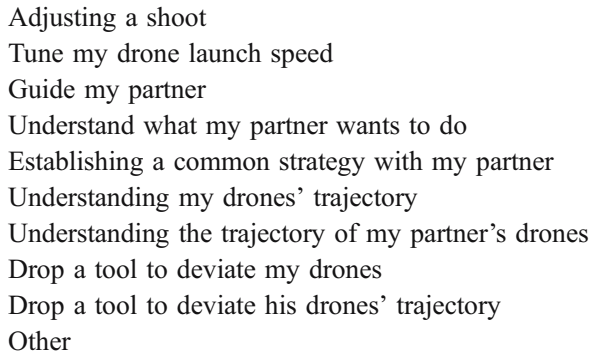

\subsection{Hypotheses}

We postulate three hypotheses:

Hypothesis H1: Pairs with awareness tools are more effective than pairs without awareness tools. The information brought by the AT should enable players to complete the task more efficiently. In order to evaluate the performance, we use the team score. We predict that the team score is higher when players have an awareness tool.

Hypothesis H2: Pairs with awareness tools build more accurate model than pairs without awareness tools. The global accuracy of the mutual modeling evaluation (MMg) should be higher when the players have an awareness tool.

Hypothesis H2: the mutual modeling accuracy improves with time: as partners learn to know each other, the representation of each others' strategies is more accurate. As a consequence, we expect MM3 to be higher than MM1.

In addition, we would like to test the correlation between the two individual mutual modeling evaluations (of the two players): is there a correlation between the objective evaluation of how B estimates A's intentions during the three events and the objective evaluation of how A estimates B's intentions during the three events? We also want to see if there is a difference in those correlations between the pairs that are provided with an AT and the pairs which are not.

Table 4 Questions asked in the second mutual modeling questionnaire (displayed after the first one) in each of the three games
What do you think your partner intend to do now?

\footnotetext{
Adjusting a shoot

Tune my drone launch speed

Guide me

Understand what I want to do

Establishing a common strategy with my partner

Understanding his drones' trajectory

Understanding my drones' trajectory

Other
} 


\subsection{Participants, settings and procedure}

Participants were all students recruited in Geneva. We chose only men in order to avoid gender bias. Experimental subjects were all familiar with computer and video games; they consisted of 18 pairs $(N=18)$. These pairs were assigned to one of the experimental conditions forming two groups of nine pairs. Participants were assigned a partner they were not familiar with. The game was played on two computers (866 MHz Intel Pentium III systems equipped with $256 \mathrm{MB}$ of RAM and ATI Radeon 8500 graphic cards) over the local network. Each player sat in front of a distinct computer located in different rooms and could communicate by voice thanks to headset. Experiments lasted approximately $2 \mathrm{~h}$ and were conducted in French. After a tutorial, players had to complete three games. mutual modeling questionnaires were displayed during missions 1, 2 (which can be completed by using two gates) and 3 (which can be completed by using two gates and a blackhole). Players had $30 \mathrm{~min}$ to complete each game level.

\section{Results}

Most of the pairs (15) played the game for the entire two h. Only three pairs managed to finish the three levels of the game in less than two h. Overall, as attested by open interviews at the end of the session, most of the players liked the game. On the whole, they were all motivated; this could be due to the fact that we selected participants who like to play computer games and were used to joysticks. They did not find the questionnaires to be disruptive since they took just few minutes to answer.

\subsection{The three hypotheses}

According to hypothesis H1, the awareness tool enables pairs to increase their performance. Table 5 shows the descriptive statistics.

The descriptive statistics shows that pairs with AT reached higher score than the others. Besides, no pairs without AT reached the mean score of the pairs with AT. the ANOVA test confirms that there is a significant difference between the condition "with AT" and "without AT" $(F=4.84, p=0.043)$. Therefore, our first hypothesis is validated: the awareness tool significantly enhances task performance.

Our second hypothesis $\mathrm{H} 2$ is that the use of an awareness tool improves the mutual modeling accuracy of a pair. We assume that players with awareness tool have more accurate mutual modeling. Table 6 shows the descriptive statistics.

Table 6 shows that the MMg (global evaluation of the mutual modeling accuracy) means are very close. This result goes against our hypothesis and the ANOVA test shows that $\mathrm{H} 2$ is invalidated $(F=0.02, p=0.889)$. The representation of one's partner strategy seems not to be facilitated by the information conveyed by the awareness tool.

Table 5 Descriptive statistics for the first hypothesis

\begin{tabular}{lllllll}
\hline & Number & Mean & Std. deviation & Min & Max \\
\hline Score & With AT & 9 & 258.67 & 90.80 & 127 & 360 \\
& without AT' & 9 & 175.67 & 67.48 & 51 & 235 \\
& Total & 18 & & & 51 & 360 \\
\hline
\end{tabular}


Table 6 Descriptive statistics for the second hypothesis

\begin{tabular}{lllllll}
\hline & & Number & Mean & Std. deviation & Min & Max \\
\hline MMg & With AT & 9 & 1.63 & 0.48 & 1 & 2.67 \\
& without AT $^{\prime}$ & 9 & 1.58 & 0.87 & 0.83 & 3.17 \\
& Total & 18 & & & 0.83 & 3.17
\end{tabular}

Our third hypothesis assumes that there is an effect of time and collaboration on mutual modeling. At the beginning of the game, the players were not familiar with each other. We hence postulated that playing together during $2 \mathrm{~h}$ would enable them to improve the accuracy of their mutual modeling. We assume that MM3, which is the mutual modeling index measured in game 3, is higher than MM1 in game 1.

As Fig. 4 illustrates, the accuracy of mutual modeling rises a little between the two first evaluations (MM1 and MM2) and then there is a sudden increase between the two last evaluations (MM2 and MM3). As a clue to this phenomenon, we should look at the moment of the evaluation of the mutual modeling accuracy. In fact, the first measure (done with the first questionnaire) appears $5 \mathrm{~min}$ after the beginning of game 1 . At this time, the players do not know each other very well since they have only played half an hour together. Thus, with the first questionnaire we have just evaluated a baseline for the accuracy of the pairs' mutual modeling. MM1 represents the evaluations of the two partners' representations of each other's strategies when the players are not very familiar with each other. The questionnaire in games 2 and 3 appears after one of the player drops an object in space. According to the log files, this event (and hence the display of the questionnaire) occurs 2 or $3 \mathrm{~min}$ after the beginning of the game. Consequently, MM2 (the evaluations of the accuracy of the mutual modeling measured in game 2) represents the MM accuracy just after the tutorial and the first game. By the same token, MM3 (the evaluations of the accuracy of the mutual modeling measured in game 3) represents the MM accuracy just after the tutorial and games 1 and 2. The implication is that perhaps the players need the tutorial and two game levels (nearly $1 \mathrm{~h}$ and a half) to be familiar with each other. The surge between MM2 and MM3 might be due to this phenomenon. Table 7 shows the descriptive statistics.

The descriptive statistics shows a slight difference between the means of MM1 and MM3. An ANOVA shows that the effect of time on the accuracy of mutual modeling is only a trend $(F=3.189, p=0.084)$ as depicted on Fig. 4 . We reject H3. Additionally, there is no effect of the presence of the awareness tool $(F=0.105, p=0.748)$. This result is consistent with the invalidation of our second hypothesis. There is also no interaction

Fig. 4 Evolution of the mutual modeling accuracy during game 1 to 3 . The horizontal axis expresses time

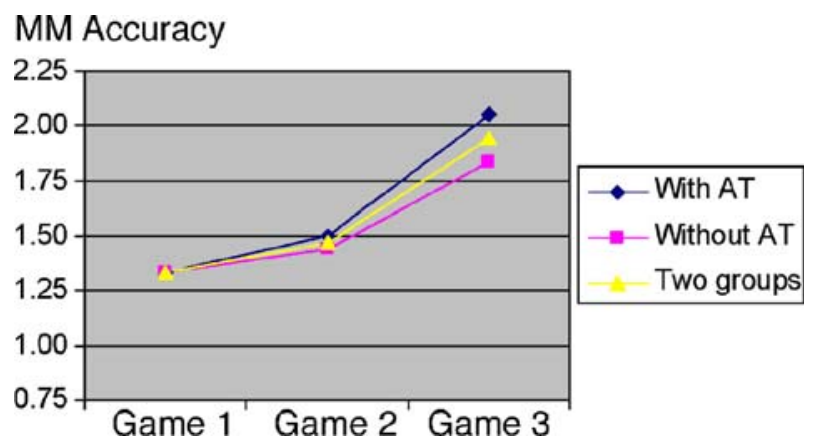


Table 7 Descriptive statistics for the third hypothesis

\begin{tabular}{lllllll}
\hline & & $N$ & Mean & Std. deviation & Min & Max \\
\hline MM & MM1 & 18 & 1.33 & 0.84 & 0 & 3 \\
& MM3 & 18 & 1.94 & 1.14 & 0.5 & 4 \\
& Total & 36 & & & 0 & 4
\end{tabular}

$M M$ Mutual modeling

between time and the presence of the awareness tool $(F=0.105, p=0.748)$. As a consequence, this supports the argument that the sudden increase of the accuracy of the mutual modeling is not due to the presence of the awareness tool.

\subsection{Additional exploratory results}

\subsubsection{Correlation between the individual mutual modeling indexes}

We investigated if there was a correlation between the objective evaluation of how player A estimates player B's strategies (calculated thanks to the results of the in-game questionnaires and compared to B's answers) and the objective evaluation of how player B estimates player A's strategies (calculated thanks to the results of the in-game questionnaires and compared to A's answers).

Results in Table 8 revealed a relatively weak positive correlation $(r=0.38)$ between the representation $\mathrm{B}$ made about $\mathrm{A}$ and the representation $\mathrm{A}$ made about $\mathrm{B}$. mutual modeling appears to be rather "a personal thing" than an activity carried out by the pair to a certain extent. As a matter of fact, if the mutual modeling was really based on the quality of the two partners interactions, the correlation would be bigger. And it is not the case, mutual modeling is hence rather a personal attitude (in the sense that people try to estimate his partner's strategies) than a reflect of the quality of the interaction between the two players in a pair.

If we consider the presence of the awareness tool, the correlation is bigger for players who did not use the AT $(r=0.44)$ than for the others who used it $(r=0.24)$. Perhaps this result could be explained by the fact that as the players without AT had less information about each other, they were forced to communicate much more and to be more explicit.

\subsubsection{Behavioral data}

To detail the analysis of the collaboration process, we focused on problem solving actions that were stored in the log files. The point was to analyze if the awareness tools changed the way users played. We counted the number of events performed by players and the whole team. We looked specifically at the number of launched drones, the number of drones docked to the space station, the number of spatial objects dragged and dropped into space, and finally the number of view change (spaceship/scout). On average, it appears that those numbers of events are quite similar in the two conditions (with awareness tools and without) as presented in Table 9. A student test was also

Table 8 Pearson bivariate correlation between the individual mutual modeling indexes of each participant in the different conditions
Pearson correlation

Player with awareness tool

0.24

Player without awareness tool

0.44 All
0.38 
Table 9 Number of events for each pair in the two conditions, standard deviation and student test findings

\begin{tabular}{llllll}
\hline & $\begin{array}{l}\text { Drones } \\
\text { launched }\end{array}$ & $\begin{array}{l}\text { Drones } \\
\text { docked }\end{array}$ & $\begin{array}{l}\text { Spatial objects } \\
\text { dropped }\end{array}$ & $\begin{array}{l}\text { Spatial objects } \\
\text { dragged }\end{array}$ & $\begin{array}{l}\text { View } \\
\text { changed }\end{array}$ \\
\hline Mean (With AT) & 416 & 70.11 & 14.44 & 14.67 & 384.67 \\
Mean (without AT) & 514.11 & 75.67 & 14.67 & 15.78 & 317.78 \\
Std Dev. (with AT) & 90.80 & 60.29 & 5.98 & 7.14 & 141 \\
Std dev. (without AT) & 280.84 & 57.28 & 4.47 & 4.60 & 176 \\
Sig. (Student test) & 0.48 & 0.84 & 0.93 & 0.70 & 0.39 \\
\hline
\end{tabular}

conducted to see if there was a difference. This test showed no significant differences between the two conditions.

Thus, there is no difference concerning the actions performed by the pairs. Players without AT changed their view less than the players in the AT condition. Players in the awareness tool condition seem to change their view a bit more often than players in the other condition, but it just a trend and it is quite logical. There is also no correlation between the number of view changes and the score $(r=0.05)$ and no correlation between the number of view changes and the accuracy of the mutual modeling as well $(r=-0.13)$.

However, if we look at the percentage of time spent in each view by the pairs in the two conditions as shown in Table 10, we notice an interesting difference. Teams in the awareness condition spent more time in the "scout view" than teams in the other condition. However, the average time spent in the "spaceship view" is similar in the two conditions. A student test showed that the difference concerning the percentage of time spent in the "scout view" is significant ( $p=0.03$ ). Since the view of the partner's scout is the awareness tool, it is not surprising that players in the tool condition spent more time in this mode. By staying in the scout view, they could give information to their partners about their intentions and their locations.

A two-way analysis of variance conducted on contrasted groups showed that pairs in the awareness condition who spent more time in the scout mode did not reach higher score $(F=1.42, p=0.26)$ than the others. Nevertheless, the analysis of variance revealed that pairs in the awareness condition who spent more time in the scout mode reached higher levels of mutual modeling $(F=8.02, p=0.015)$ than the others. It implies that there is an effect of the awareness tool on the accuracy of the mutual modeling only for the teams who spent a long time in the scout mode. Therefore, it seems that the information conveyed by the awareness tool could be a benefit for team collaboration. Those information could help players in order to estimate their partner's strategies if the participants understand that they have to make an effort: spending an accurate time in the scout view. The team who did not spend enough time in the scout view had no benefit of the awareness tool.

Table 10 Average percentage of time spent in the "scout view" and in the "spaceship view" by the pairs in the two conditions

\begin{tabular}{lll}
\hline & $\begin{array}{l}\text { With } \\
\text { awareness } \\
\text { tool }(\%)\end{array}$ & $\begin{array}{l}\text { Without } \\
\text { awareness } \\
\text { tool }(\%)\end{array}$ \\
\hline $\begin{array}{l}\text { Average percentage of time spent } \\
\text { in the "scout view" }\end{array}$ & 75.41 & 62.54 \\
$\begin{array}{l}\text { Average percentage of time spent } \\
\text { in the "spaceship view" }\end{array}$ & 24.59 & 37.46 \\
\hline
\end{tabular}




\section{Discussion}

We explored the field of research concerning the cognitive impacts of the awareness tools used in collaborative multi-user environments. The study we have presented in this document addresses two issues: the effect of the awareness tool on performance and the impact on the mutual modeling. So far, only some findings confirmed our hypotheses and intuitions. Some results, though seem to be contrary to expectations.

The fact that teams in the awareness tool condition reach higher score than the others is consistent with the findings of [14] and [7]. They also found that awareness could be beneficial to team performance. In fact, this tool provides a continuous feedback to the partner who can see where is his teammate. This could be extremely useful in tasks like object positioning. In such tasks, player A guided player B's movement by giving him instructions about where dropping the object. Additionally, the AT provided visual evidences about the player's location (in scout or explorer). The team was thus more effective because player A had not to verbally describe where he was and player B had not to interpret this description. As suggested by [14], the use of the awareness tool leads to transform a task from a verbal to a visual activity.

Our second hypothesis was invalidated: the representation of one's partner strategy is not facilitated by the information conveyed by the awareness tool. Presumably, those results lead us to four possible conclusions. First, the awareness tool, by showing information about the partner's locations and gaze does not improve the accuracy of the mutual modeling. One reason could be that the information conveyed by the AT is not useful for that task. Second, it is possible that the game does not require participants to maintain accurate mutual models; they do not have to care much about each other. Third, the task could have been so difficult that players did not have enough time or attention to spare for the awareness tool. And finally, perhaps, our evaluation of mutual modeling is not very precise.

We also focused on behavioral data that were stored in the log files. Looking specifically at the percentage of time spent in each view (scout or spaceship) by the pairs; we could notice an interesting difference. A two-way analysis of variance conducted on contrasted groups showed that pairs in the awareness condition who spent more time in the scout mode reached higher levels of mutual modeling $(F=8.02, p=0.015)$ than the others. It implies that there is an effect of the awareness tool on mutual modeling only for the teams who spent a long time in the scout mode. Awareness information could help players in order to estimate their partner's strategies if the participants understand that they have to make an effort: spending an accurate time in the scout view. The team who did not spend enough time in the scout view had no benefit of the awareness tool. Our finding raises a new question: do players really use the awareness tool? Indeed, if there is an effect of the tool on mutual modeling only for the players who used it frequently, it may be possible that only a few players in the tool condition noticed the advantage of using it.

Hypothesis 3 postulated an effect of time on the accuracy of mutual modeling. There seems to be an effect but it is not statistically significant. Our hypothesis is invalidated but the findings call for certain restrictions because of the questionnaire. First, the questionnaire per se could be disruptive and could push players to pay more attention to their partner's intents. Second, the questions we asked are limited in the sense that it just refers to a specific moment in the game and not to real actions.

Therefore, generalizing these results to other contexts than this video game, we found that:

- Awareness tools could ease task performance if they provide a good way to support referential communication among teammates. A condition for this is that the AT is 
properly matched to the task. The tool should indeed make sense in the context of the task performed; for instance there should be objects or artifacts to describe and for which the awareness tool allow to reference.

- Awareness tools, if their meaning and use is properly understood, could help people to get more insights about their partner's intents. The condition for this result is indeed that the users get the added value of such a tool. Noticing the gain due to the awareness information is not obvious per se since the users have first to understand the task to be performed and which kind of tool could support (a) the task performance and (b) the collaboration.

Nonetheless, those results show certain limitations. On the one hand, the number of participants is quite low: 18 pairs (nine in each conditions). On the other hand, the method used to measure the accuracy of the mutual modeling may be unsuitable. Using a simple questionnaire to measure the accuracy in predicting partner's answers is way too subjective. We should use a more objective method to evaluate this variable. A solution would be to compare what player A thinks B is going to do with what B really does.

We could criticize the tool we used as well. We considered awareness within a simple tool that conveys specific information about the participants' behavior. In fact, awareness is more than the information presented in this kind of "widget"; the situation is more intricate. We should reconsider the definition of awareness as a diffuse flow of information as in [22]: lots of multiple cues, signs, evidences which are all combined. This flow makes sense and it is very difficult to create a tool to enable this combination. Another major drawback of our experiment concerns the fact that we provided the users with a voice over IP communication tool. It is therefore difficult to isolate the awareness information related to the tool from those related to the voice communication. The only way we can deal with this issue is to investigate the dialogues and check where they mention awareness information related or not to the AT. A comparison of the dialogue between the two groups would be very instructive for that matter. Anyhow, in one of the pre-test we let players use the game without any communication tool and it was really impossible for them to complete the first level. This feature is mandatory in order to support the collaboration.

In order to revalidate and confirm our results, we are thinking about several tracks:

- Expanding our sets of data by having more participants in each group.

- Improving our mutual modeling analyzing tool. This could be done through an analysis of participants' action. The point would be to compare what player A thinks B is going to do with what B really does which is more precise than just asking the players what they do. We could also consider the redundancy of actions as a coordination index: if player A did the same actions as player B, that could means that they did not coordinate so much.

- We are intending to provide the players with a replay tool in order to confront them to what they did. This will allow them to have a basis to explain what happened.

- A dialogue analysis among each pairs will also be conducted, especially to see how participants without the awareness tool deal with positioning description and how the presence or the lack of the tool modified the interactions.

Moving from this game to much more generalized lessons, there are several impacts for practitioners as well, for instance for Computer Support for Cooperative Work. The findings provide evidence that location and gaze awareness can be useful in certain 
situations. As we mentioned above, the use of the awareness tool in Spaceminers leads people to transform a task from a verbal to a visual activity and hence induce a quicker completion of the task. This a clear lesson for designers: instead of letting participants describing their locations or the artifacts they are talking about, an awareness tool could usefully show those kind of indications. The AT in this context supports very well what is called referential communication, the process by which a speaker talks about an object to his addressee. Another interesting lesson is that designers should not taken for granted that users will systematically employ an AT simply because it is available. As we have seen in our experiment, several players did not really notice the potential of this tool. Thus, designers should provide users with usable AT and teach them their real value.

Acknowledgment The research carried out at the Geneva Interaction Lab was partly funded by Pictet\&Cie.

\section{References}

1. Collazos C, Guerrero L, Pino J, Ochoa S (2002) Introducing knowledge-shared awareness. In Proc. of IASTED international conference: information and knowledge sharing (IKS 2002). St. Thomas, Virgin Islands, USA, pp 13-18

2. David JMN, Borges MRS (2001) Selectivity of Awareness Components in Asynchronous CSCW Environments. In Proc. of the 9th International Workshop on Groupware, CRIWG. Darmstadt, Germany, pp $115-124$

3. Decortis F, Noirfalise S, Saudelli B (1997) Cognitive ergonomics as a framework for cooperative work. Theoretical approaches for analysing cooperative technologies for complex work settings. Report of Work Package 1, EU-TMR Network COTCOS. University of Sussex, Sussex

4. Dillenbourg P (1999) What do you mean by collaborative learning?. In: Dillenbourg P (ed) Collaborative learning: cognitive and computational approaches. Elsevier, Oxford, pp 1-19

5. Dourish P, Bellotti V (1992) Awareness and coordination in shared workspaces. In Proc. of ACM CSCW'92 conference on computer supported cooperative work. Toronto, Canada, pp 107-114

6. Ellis C, Gibbs S, Rein G (1991) Groupware: some issues and experiences. Commun ACM 34(1):38-58

7. Espinosa A, Cadiz J, Rico-Gutierrez L, Kraut R, Scherlis W, Lautenbacher G (2000) Coming to the wrong decision quickly: why awareness tools must be matched with appropriate tasks. In Proc. of the CHI Conference on Human factors in computing systems. The Hague, The Netherlands, pp 392-399

8. Fussell SR, Krauss RM (1992) Coordination of knowledge in communication: effects of speakers' assumptions about what others know. J Pers Soc Psychol 62(3):378-391

9. Gaver WW (1991) Sound support for collaboration. In Proc. of ECSCW 91. Dordrecht, The Netherlands, pp 293-308

10. Greenberg S, Gutwin C, Cockburn A (1996) Awareness through fisheye views in relaxed-WYSIWIS groupware. In Proc. of the graphics interface conference. Toronto, Canada, pp 28-38

11. Grudin J (1994) Computer-supported cooperative work: its history and participation. IEEE Computer 27 (5):19-26

12. Gutwin C, Greenberg S (1999) A framework of awareness for small groups in shared-workspace groupware. Technical Report 99-1, Department of Computer Science, University of Saskatchewan, Canada

13. Gutwin C, Greenberg S (1999) The effects of workspace awareness support on the usability of real-time distributed groupware. ACM Trans Comput-Hum Interact 6(3):243-281

14. Gutwin C, Roseman M, Greenberg S (1996) A usability study of awareness widgets in a shared workspace groupware system. In Proc. of the ACM conference on computer supported cooperative work. Boston, USA, pp 258-267

15. Heath C, Luff P (1992) Collaboration and control: crisis management and multimedia technology in london underground line control rooms. CSCW: The Journal of Collaborative Computing 1(1-2):69-94

16. Heider F (1958) The psychology of interpersonal relations. Wiley, New York 
17. Isaacs H, Tang JC, Morris T (1996) Piazza: a desktop environment supporting impromptu and planned interactions. In Proc. of ACM CSCW'96. Boston, USA., pp 315-324

18. Isaacs H, Walendowski A, Ranganathan D (2002) Hubbub: a sound-enhanced mobile instant messenger that supports awareness and opportunistic interactions. In Proc. of the Conference Computer-Human Interaction (CHI). Minneapolis, USA, pp 179-186

19. Jang CY, Steinfield C, Pfaff B (2002) Virtual team awareness and groupware support: an evaluation of the TeamSCOPE system. Int J Human-Comput Stud 56:109-126

20. Krauss RM, Fussell SR (1991) Perspective-taking in communication: the determination of others' knowledge and referential language use. Social Cogn 9(1):2-24

21. Ljungstrand P, Segerstad YH (2000) An analysis of webwho : how does awareness of presence affect written messages? In Proc. of the int. workshop on awareness and the WWW, ACM CSCW'2000 conference. Philadelphia, USA, available on-line at http://www2.mic.atr.co.jp/dept2/awareness

22. Mastrogiacomo S (2002) Utilisation des zones de travail partagées asynchrones pour améliorer la compréhension mutuelle dans les groupes de projet distribués. Ph.D. Thesis, Université de Lausanne, June

23. Matusov E (1996) Intersubjectivity without agreement. Mind Cult Act 3(1):25-47

24. Nickerson RS, Baddeley A, Freeman B (1987) Are people estimates of what other people know influenced by what they themselves know?. Acta Psychol 64:245-259

25. Ogata H, Yano Y (2000) Combining knowledge awareness and information filtering in an open-ended collaborative learning environment. Int J Artif Intell Educ 11:33-46

26. Ott D, Dillenbourg P (2002) Proximity and view awareness to reduce referential ambiguity in a shared 3D virtual environment. In Proc. of computer supported collaborative learning 2002. Boulder, Colorado, USA, pp 603-604

27. Schmidt K (2002) The problem with "awareness": introductory remarks on "awareness in CSCW". CSCW: The Journal of Collaborative Computing 11(3-4):285-298

28. Sohlenkamp M (1999) Supporting group awareness in multi-user environments through perceptualization. Technical Report No. 6, GMD Institute, Germany

29. Stefik M, Bobrow D, Foster G, Lanning S, Tatar D (1997) «WYSIWIS revised: early experiences with multi-user interfaces $\gg$. ACM TOIS 5(2):147-167

30. Stefik M, Foster G, Bobrow D, Kahn K, Lanning S, Suchman L (1987) Beyond the chalkboard: computer support for collaboration and problem solving in meetings. Commun ACM 30(1):32-47

31. Wegner DM (1987) Transactive memory: a contemporary analysis of the group mind. In: Mullen B, Goethals GR (eds) Theories of group behavior. Springer, Berlin Heidelberg New York, pp 185-208

32. Wertsch JV (1985) Vygotsky and the social formation of mind. Harvard University, Cambridge, MA

33. Won M, Pipek V (2004) Sharing knowledge on knowledge-the eXact peripheral expertise awareness system. J UCS 9(12):1388-1397

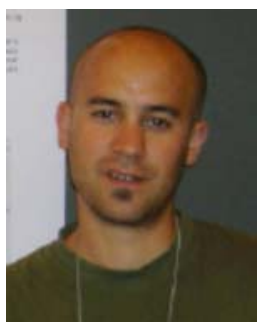

Nicolas Nova received an undergraduate degree in cognitive sciences from the University of Lyon, France and a M.Sc in human-computer interaction from the University of Geneva, Switzerland. He is now a Ph.D student at CRAFT, an educational technologies and HCI lab, at the Swiss Institute of Technology in Lausanne (EPFL). The focus of his research is the empirical study of technology usage, from a sociocognitive point of view. His current projects is headed towards the investigation of how location-awareness influence collaboration processes in mobile settings. He is also doing consulting work for European companies about user experience research in different field ranging from video games to web platforms. 


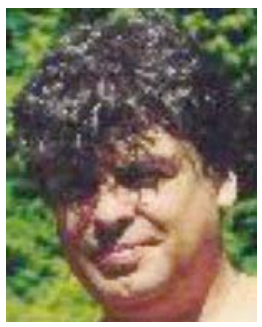

Thomas Wehrle studied Psychology and Computer Science at the University of Zürich. From 1989 to 1992 he was a research assistant at the Artificial Intelligence Lab of the Computer Science Department at the University of Zurich. He finished his Ph.D. in 1993 on psychological modeling and simulation of autonomous agents. He was a research fellow and principal researcher in several NSF projects in the Geneva Emotion Research Group and the Geneva Interaction Lab at the University of Geneva. Since 2004 he is a senior research fellow at the Psychological Methods Group at the University of Zurich. His main research interests concern emotional problem solving, modeling and expression of emotion, collaboration, experimental platforms and micro worlds for experimental research, and new technologies.

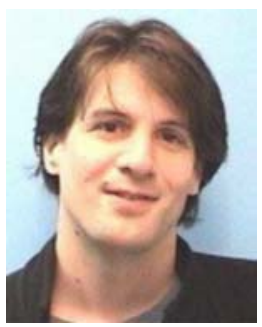

Jeremy Goslin received a B.Eng(Hons) from Staffordshire University, UK, an M.S. from Essex University, and $\mathrm{Ph} . \mathrm{D}$ in computer science from Sheffield University. He subsequently worked at the University of Geneva, Tufts University, Boston, and the University of Aix-en-Provence, France, before joining the School of Psychology at Plymouth University, UK, as a research fellow. His current research interests are in the area of cognitive psychology, with special interest in language production deficits due to cognitive aging.

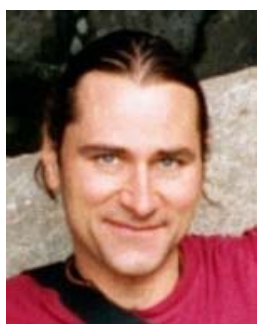

Yvan Bourquin has a BSc degree in Software Engineering from the University of Berne and an MSc in Evolutionary and Adaptive Systems from the University of Sussex, UK. He has also worked in the field of Computer Aided Surgery (CAS) at the University of Berne and in the field of human computer interactions and information visualization at the University of Geneva. He currently works at the EPFL in Lausanne as a software developer on a project aiming at extending and adapting the Webots robot simulator to educational environments. 


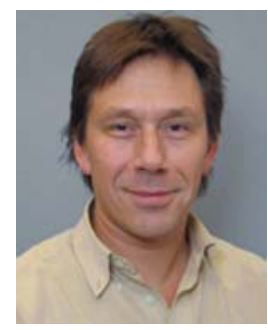

Pierre Dillenbourg former teacher in elementary school, Pierre Dillenbourg graduated in educational science (University of Mons, B). He started his research on learning technologies in 1984. He obtained a Ph.D. in computer science from the University of Lancaster (UK), in the domain of artificial intelligence applications for educational software. He has been director of TECFA, the educational technology unit at the University of Geneva. He joined EPFL in November 2002. His current interests concern computer-supported collaborative learning: the role of virtual space in social interaction; the effects of awareness tools on group performance and mutual modeling; the design of mixed-reality learning environments; mobile technologies for learning. He is also a consultant working on training technologies and HCI issues for large companies in Switzerland and Europe. 